\title{
What's New in Salvage of the Ischemic Myocardium: Estrogen Postconditioning
}

\author{
Fawzi A. Babiker Shaji Joseph Jasbir S. Juggi \\ Department of Physiology, Faculty of Medicine, Health Sciences Centre, Kuwait University, Jabriya, Kuwait
}

\section{An Early Intervention in the Treatment of Ischemic Heart Disease}

Ischemia-reperfusion injury, infarct size and the prognosis of ischemic heart disease have been challenges for cardiovascular scientists and cardiologists for decades. After extensive research and trials, scientists introduced the technique of reopening an occluded blood vessel for reperfusion of the infarcted area. This technique has saved many lives, and progression of ischemic heart disease has started to decline. Nevertheless, many scientists have expressed concern about myocardial damage caused by reperfusion itself, later identified and described as reperfusion injury. This injury has been a major setback in the use of reperfusion as a treatment. As a solution, scientists started to replace the first complete opening of the occluded blood vessel with slow or graded reperfusion of the organ concerned.

\section{Ischemic Pre- and Postconditioning}

While success of the technique approached its zenith in clinical practice, the search continued for an effective solution to the proliferation of ischemic heart disease. A pioneer in the field of ischemic reperfusion research,
Murry et al. [1], devised the promising technique of preconditioning to salvage the myocardium from perfusion's subsequent ischemic insult. This technique is used for repetitive brief episodes of ischemia before the occurrence of prolonged major ischemia that becomes lethal. While the technique is very promising as a new method of treatment for ischemic heart disease, its application in clinical practice has been arduous and highly risky. The difficulty is caused by a lack of the means to determine the time of occurrence of ischemia. Nevertheless, the technique can be applied in open heart surgery. At this level, repetitive inflating and deflating of the angioplasty balloon is easy to apply with almost no damage to the occluded blood vessel and can offer salvage from subsequent prolonged ischemia. Sometime later, Vinten-Johansen and his coworkers [2] introduced a technique similar to preconditioning with a greater probability of application to actual clinical practice. Postconditioning, the newly introduced technique, is the application of brief, repetitive ischemia and reperfusion. However, the innovation is that the procedure is performed after the ischemic insult and immediately at the beginning of reperfusion. This technique was found to be very efficient in providing protection to the ischemic myocardium and easily applied in clinical practice.

\section{KARGER}

Fax +41613061234

E-Mail karger@karger.ch

www.karger.com
(C) 2011 S. Karger AG, Basel

1011-7571/11/0206-0495\$38.00/0

Accessible online at:

www.karger.com/mpp 


\section{Remote Pre- and Postconditioning}

Because preconditioning is difficult to translate to actual clinical practice, remote preconditioning was introduced by Przyklenk et al. [3]. This technique is a repetitive transient ischemia on a remote specific organ or vascular bed far from the insulted organ, but leads to its protection from the subsequent prolonged ischemic reperfusion damage, the same principle as inducing transient ischemia in a limb, which subsequently limits the extent of the infarcted myocardium. This technique showed encouraging results in small clinical studies. Another method of protection similar to remote preconditioning was introduced by Botker et al. [4]. This technique is similar to classical postconditioning, but is only applied in a remote organ or artery at the beginning of the ischemic challenge. This method of protection is safer than classic postconditioning. One of the interesting methods used in this technique is repetitive inflation and deflation of the blood pressure cuff before reperfusion or even during the transportation of the patient to the hospital. Remote postconditioning is considered to be a promising novel approach in the salvage of the ischemic myocardium, but it is still in an early stage of development. If successful, it will result in easy control of ischemia-reperfusion injury without the risk of involving coronary circulation in the process.

\section{Future of Estrogen in Postconditioning Research}

Estrogen is recognized to play a significant role in the protection of the heart against cardiovascular diseases, but the exact mechanism is still controversial. Estrogen treatment has been shown to play a very important role in the salvage of ischemic myocardium after challenging ischemic events in studies of estrogen protection to the heart. The advances attained in the field of conditioning necessitate introduction of noninvasive techniques and drugs in conditioning research. Surprisingly, introduction of pharmacological drugs in conditioning research is moving very slowly, encumbered by disappointing results in earlier large-scale experiments. Filling in the gaps and enhancing drug use in conditioning estrogen will be two areas for further study, since those done on the use of estrogen in preconditioning and postconditioning have been very few, but with encouraging results. Estrogen research in this field is urgently required, as most of conditioning intervention in ischemic heart disease is influenced by the presence or absence of estrogen and gender differences. Introducing estrogen in postconditioning research seems to be a suitable technique if some concerns about its use are addressed. Estrogen and other pharmacological agents might also be suitable candidates for postconditioning research when the required developments for its application in clinical practice are achieved. Finally, estrogen research is required to determine the safest methods of use in postconditioning protection for male and female patients. Moreover, future work on the mechanisms of estrogen protection and its downstream pathways will add much to understanding the mechanisms of postconditioning protection. Some protective elements selected from the uncovered protection cascade or the protective metabolites of estrogen can be used for myocardium protection. They can also be combined pharmacologically with other postconditioning methods to attain the maximum required protection.
References
1 Murry CE, Jennings RB, Reimer KA: Preconditioning with ischemia: a delay of lethal cell injury in ischemic myocardium. Circulation 1986;74:1124-1136.

-2 Zhao ZQ, Corvera JS, Halkos ME, Kerendi F, Wang NP, Guyton RA, Vinten-Johansen J: Inhibition of myocardial injury by ischemic postconditioning during reperfusion: comparison with ischemic preconditioning. Am J Physiol Heart Circ Physiol 2003;285:H579H588.

\footnotetext{
3 Przyklenk K, Bauer B, Ovize M, Kloner RA, Whittaker P: Regional ischemic 'preconditioning' protects remote virgin myocardium from subsequent sustained coronary occlusion. Circulation 1993;87:893-899.

-4 Botker HE, Kharbanda R, Schmidt MR, Bottcher M, Kaltoft AK, Terkelsen CJ, Munk $\mathrm{K}$, Andersen NH, Hansen TM, Trautner S, et al: Remote ischaemic conditioning before hospital admission, as a complement to angioplasty, and effect on myocardial salvage in patients with acute myocardial infarction: a randomised trial. Lancet 2010;375:727-734.
} 